\title{
Antihypertensive drug prescription patterns and their impact on outcome of blood pressure in Ethiopia: a hospital-based cross-sectional study
}

This article was published in the following Dove Press journal:

Integrated Pharmacy Research and Practice

27 January 2017

Number of times this article has been viewed

\author{
Tadesse Melaku Abegaz \\ Yonas Getaye Tefera \\ Tamrat Befekadu Abebe \\ Department of Clinical Pharmacy, \\ School of Pharmacy, College of \\ Medicine and Health Sciences, \\ University of Gondar, Gondar, \\ Ethiopia
}

\begin{abstract}
Background: Irrational prescription is strongly associated with poor control of hypertension. The present study aimed to evaluate antihypertensive drug prescription trends and to measure their impact on the level of blood pressure (BP) control in Gondar University Hospital, Gondar, Ethiopia.

Methods: A hospital-based retrospective cross-sectional study was conducted from May 30 to June 30, 2016. All hypertensive patients on medication were included. A structured data abstraction form was prepared to gather the necessary information. The prescription patterns and BP level were measured retrospectively. A binary logistic regression was computed to determine the effect of different prescription patterns on BP control.

Results: A total of 596 hypertension patients were recruited for the study; of them, 561(94\%) met the study criteria. The mean age of the respondents was $55.96 \pm 14.6$ years. Females constituted $58.2 \%$ of the study population. Approximately fifty percent of the prescriptions were monotherapies. Twice-daily dosing was associated with lower risk of uncontrolled hypertension (crude odds ratio $[\mathrm{COR}]=0.51[0.15-0.73]$, adjusted odds ratio $[\mathrm{AOR}]=0.69[0.163-0.91]$ ) Monthly appointment was linked with a nearly $90 \%$ reduced incidence of uncontrolled BP $(\mathrm{COR}=0.15[0.04-0.73]$, AOR $=0.093[0.024-0.359])$.

Conclusion: Monotherapies were the most frequently prescribed regimens. Twice-daily dosing and monthly appointments were associated with low incidence of uncontrolled BP. Clinicians should be vigilant in adjusting the frequency of dosing and should fix appointment date in consultation with their patients.
\end{abstract}

Keywords: prescription patterns, blood pressure, Ethiopia

\section{Introduction}

Noncommunicable diseases are a growing public health concern, particularly hypertension (HTN), with an appreciable proportion of patients with HTN living in Africa. HTN is a chronic form of illness that requires lifelong medications. There are different pharmacological approaches to the management of HTN including diuretics, $\beta$-blockers, calcium channel blockers, and renin-angiotensin inhibitors. Each of the classes of medications have their own indication. ${ }^{1}$ Angiotensin-converting enzyme inhibitors (ACEIs) are especially important in patients with diabetes mellitus (DM) as they can help prevent nephropathy. To achieve the ultimate goal of treatment of HTN, antihypertensives should be prescribed rationally. ${ }^{2,3}$

Rational prescription patterns are defined as patients receiving medications according to their medical condition, in doses that meet their requirements, for an adequate
Correspondence: Tadesse Melaku Abegaz Department of Clinical Pharmacy, School of Pharmacy, College of Medicine and Health Sciences, University of Gondar, PO Box 196, Gondar, Ethiopia Tel +25I 942857975

Email melakutadesse98@gmail.com 
period of time, and at affordable cost to them and the society. Appropriate prescribing has a positive implication on medication adherence and disease improvement. ${ }^{4}$ Irrational prescription pattern (IPP) occurs when the antihypertensive drug is given at inappropriate dose, frequency, and duration. Mao et $\mathrm{al}^{5}$ stated that about $60 \%$ of medicines in public health facilities and $70 \%$ of medicines in private health cares were prescribed and sold inappropriately in developing countries. IPP is strongly associated with poor control of chronic diseases including HTN. Uncontrolled blood pressure (UBP) is an independent risk factor leading to enormous complications and mortalities secondary to HTN. Inappropriate provision of medications could also lead to extra expense incurred to the patient due to ineffective treatment and adverse drug reactions. ${ }^{6}$ Copayment for antihypertensives might also contribute for poor disease outcome. ${ }^{7}$ The lack of improvement and appearance of untoward side effects with the prescribed medication could result in distrust between the patient and the clinician ${ }^{8,9}$ Infective drugs also provoke the physicians to frequently change the regimen and dose, thereby leading to fluctuation in patients' BP. ${ }^{10}$ The elderly are usually prone to experiencing adverse drug events from irrational prescription as their drug excreting and metabolizing organs have atrophied over time (as a result of aging). ${ }^{11}$ In addition, many older patients also have comorbidities and consume multiple drugs. ${ }^{12}$ Polypharmacy was found to be associated with high prevalence of IPP. $^{13}$

As a component of pharmacoepidemiological techniques, prescribing pattern surveys provide an unbiased picture of prescribing habits and help identify suboptimal prescribing patterns for further evaluation. Such kinds of analytical studies are the best design to compare drug utilization and prescription patterns with respect to outcome of treatment. Prescribing pattern surveys of chronic medication could be assumed as medication reconciliation (Medrec) if they can be carried out periodically. Chiatti et al $^{12}$ stated that routine evaluation of prescription patterns resulted in a cost saving of more than USD307.4 million per year.

The effect of prescription patterns on the control of BP has not been rigorously studied in north Ethiopia in the outpatient setting. Few studies were performed on psychotropic, narcotic, and anticonvulsant drugs, ${ }^{14}$ while others were done on inpatient pediatric populations. ${ }^{15}$ These studies also did not measure the effect of IPP on treatment outcome. The present study particularly aimed to compare the impact of different antihypertensive drug prescription trends on the level of BP control in Gondar University Hospital, Gondar, Ethiopia.

\section{Methodology}

Study setting

This study was conducted at Gondar University Hospital, which is located in northwestern part of Ethiopia. It serves more than 5 million people in the area. The hospital contains outpatient, inpatient, pediatric, emergency, and ophthalmology pharmacies. As it is a referral hospital, it contains different classes and dosage forms of medicines that can be used for the treatment of both chronic and emergency forms of HTN. Some patients do have free access (insured) to medications.

\section{Study design}

A hospital-based retrospective cross-sectional study was conducted from May 30 to June 30, 2016 in the Gondar University Hospital.

\section{Study population}

Patient cards of all HTN patients that were available during the study period were evaluated. Around 561 patients met the inclusion criteria. Patient cards with incomplete data on duration, dose and frequency, and patients on lifestyle modification and BP not taken for further consideration. Patients who were transferred, lost to follow-up, or died were also removed from the study population.

\section{Variables}

BP was our dependent variable. Sex, residence, dose, duration of appointment, frequency, drug interaction, drug selection, and regimen change were the independent variables.

\section{Operational definitions}

\section{Irrational prescription}

It is defined as the ordering of inappropriate drug with incorrect dose, frequency, and duration without considering the cost incurred to the patient or to the community at large. It was measured by reviewing patient medical records and comparing the pattern with national and international clinical practice guidelines (CPGs; ie, standards). ${ }^{3,16,17}$

\section{Data collection}

Data were collected by investigators working at the Department of Clinical Pharmacy, University of Gondar as instructors and clinical pharmacists. A data abstraction form was prepared to gather the necessary information. It contained items related to dose, frequency, duration, drug class and 
interaction, and $\mathrm{BP}^{18} \mathrm{BP}$ level was taken from patients' medical record during their last visit. Drug-related problems (DRPs) were compiled from prescriptions and further assessed using practice guidelines. Rate of BP control was estimated by the number of patients who attained goal BP. It was set below 140/90, with a special cut-off point. The proportion of patients on monotherapy, dual therapy, and triple therapy was subsequently calculated.

\section{Data analysis}

Data were entered into and analyzed using SPSS software version 20 (IBM, Armonk, NY, USA) for Windows. Descriptive statistics including frequency, percentage, mean, and standard deviation were used for describing sociodemographic characteristics. Binary logistic regression was computed to determine the effect of irrational prescription on BP control. A $p$-value of $<0.05$ was regarded as statistically significant in the regression models with $95 \%$ confidence interval to test the level of significance.

\section{Ethics}

Ethical approval was obtained from the ethical committee of the School of Pharmacy, College of Medicine and Health Sciences, University of Gondar for this study. Patient consent was deemed not required by the ethical committee of the University of Gondar due to anonymised data and the retrospective nature of this study.

\section{Results}

\section{Patient characteristics}

A total of 596 HTN patients were recruited for the study; of them, 561(94\%) met the study criteria and were included. The mean age of the respondents was $55.96 \pm 14.6$ years. Their mean BP was 138/87 mmHg. More than one-half of the study population was female (58.2\%). Nearly two-thirds of patients were above 50 years of age $(67.4 \%)$. Approximately one-third of the participants were living with other comorbidities 157(28\%), with DM (33.1\%) ranking first followed by cardiovascular diseases $27(17.2 \%$ ) (Table 1).

\section{DRPs among hypertensive patients}

More than one-half of the respondents experienced DRPs, 292(52\%). The most frequent DRPs was "dose too low" 103(35.27\%). The least common DRP was "needs additional drug therapy" 16(5.47\%) followed by "unnecessary drug therapy" 19(7.87\%) (Table 2).

There were statistically significant variations in the frequency of DRPs among patients with controlled and UBP. Spe-
Table I Baseline characteristics of respondents

\begin{tabular}{lll}
\hline Variables & Variables & Frequency $\mathbf{n}(\%)$ \\
\hline Sex & Female & $327(58.2)$ \\
Age (years) & Male & $234(41.8)$ \\
& $21-30$ & $30(5.3)$ \\
& $31-40$ & $66(10.7)$ \\
& $41-50$ & $86(15.3)$ \\
& $51-60$ & $143(25.5)$ \\
Residence & $61-70$ & $133(23.7)$ \\
& $70+$ & $102(18.2)$ \\
Comorbidities & Urban & $430(76.6)$ \\
& Rural & $131(23.3)$ \\
& DM & $52(33.1)$ \\
& Asthma & $16(10.2)$ \\
& Dyslipidemia & $22(14.0)$ \\
& Arthritis & $18(11.5)$ \\
& PUD & $16(10.2)$ \\
& Cardiovascular & $27(17.2)$ \\
& Others & $6(3.8)$ \\
\hline
\end{tabular}

Abbreviations: $n$, number of patients; DM, diabetes mellitus; PUD, peptic ulcer disease.

Table 2 The types of DRPs with respect to the level of BP control

\begin{tabular}{|c|c|c|c|c|c|}
\hline DRPs & Controlled & Uncontrolled & DRPs & U-test & $p$-value \\
\hline & BP n (\%) & BP n (\%) & (Total, n) & & \\
\hline $\begin{array}{l}\text { Unnecessary } \\
\text { drug therapy }\end{array}$ & $8(34.78)$ & $15(65.22)$ & 23 & 8.4 & $0.0 \mathrm{I}^{\mathrm{a}}$ \\
\hline $\begin{array}{l}\text { Needs } \\
\text { additional } \\
\text { drug }\end{array}$ & $5(31.25)$ & II (68.75) & 16 & 13.9 & $0.02^{\mathrm{a}}$ \\
\hline $\begin{array}{l}\text { Ineffective } \\
\text { drug therapy }\end{array}$ & II(57.89) & $8(42.11)$ & 19 & 17.5 & 0.45 \\
\hline $\begin{array}{l}\text { Dose too } \\
\text { low }\end{array}$ & $54(52.43)$ & $49(47.57)$ & 103 & 9.3 & 0.06 \\
\hline $\begin{array}{l}\text { Dose too } \\
\text { high }\end{array}$ & $33(63.46)$ & $19(36.54)$ & 52 & 11.4 & 0.07 \\
\hline $\begin{array}{l}\text { Adverse } \\
\text { drug } \\
\text { reaction }\end{array}$ & $5 I(64.56)$ & $28(35.44)$ & 79 & 16.2 & $0.00^{\mathrm{b}}$ \\
\hline
\end{tabular}

Notes: ${ }^{2}$ Significant at 0.05 level, bsignificant at 0.01 . Nonparametric Mann-Whitney U-test.

Abbreviations: $n$, number of patients; DRPs, drug-related problems; BP, blood pressure.

cifically, "unnecessary drug therapy" was significantly noticed in patients with UBP (65.22\%). Patients with controlled BP usually suffered from adverse drug reactions (64.56\%), whereas those with UBP required additional drug therapy (68.78\%).

\section{Prescription patterns of antihypertensive medications}

Enalapril, hydrochlorothiazide, spironolactone, enalapril, frusemide, and atenolol were among the antihypertensive drugs available. Hydrochlorothiazide was the most commonly prescribed monotherapy $(60.24 \%)$ followed by enalapril 
(17.51\%).The least common single antihypertensive agent used was atenolol (1.6\%) (Figure 1).

The most common dual therapy was enalapril plus hydrochlorothiazide (53.68\%), accounting for nearly one-half of dual-therapy prescriptions. A combination of nifedipine and hydrochlorothiazide was the second most common dual combination therapy (29.44\%). Atenolol with hydrochlothiazide $(3.5 \%)$ was one of the less frequently prescribed combinations (Figure 2).

The most common triple-therapy antihypertensive medication combination prescribed was (Atenolol + hydrochlothiazide + enalapril) (39.68\%). The combination of nifedipine plus enalapril and spironolactone was rarely prescribed (11.11\%) (Figure 3).
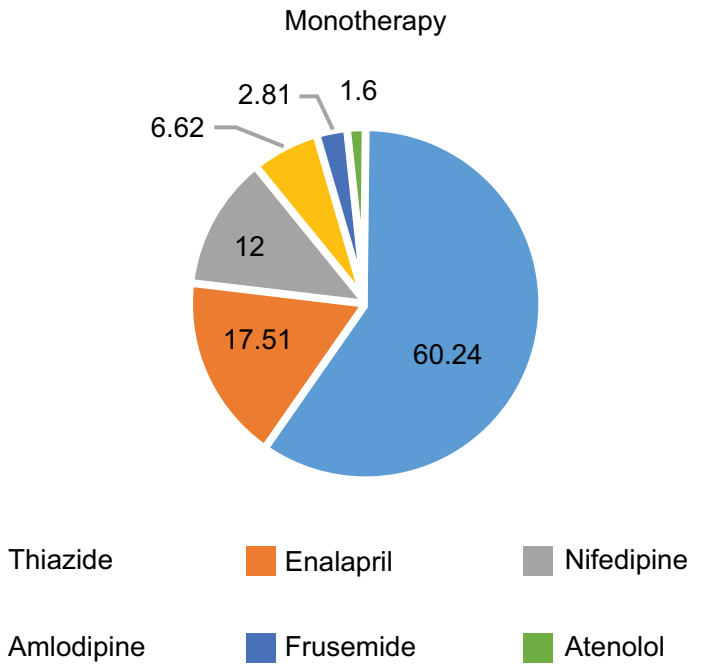

Figure I The type of monotherapies.

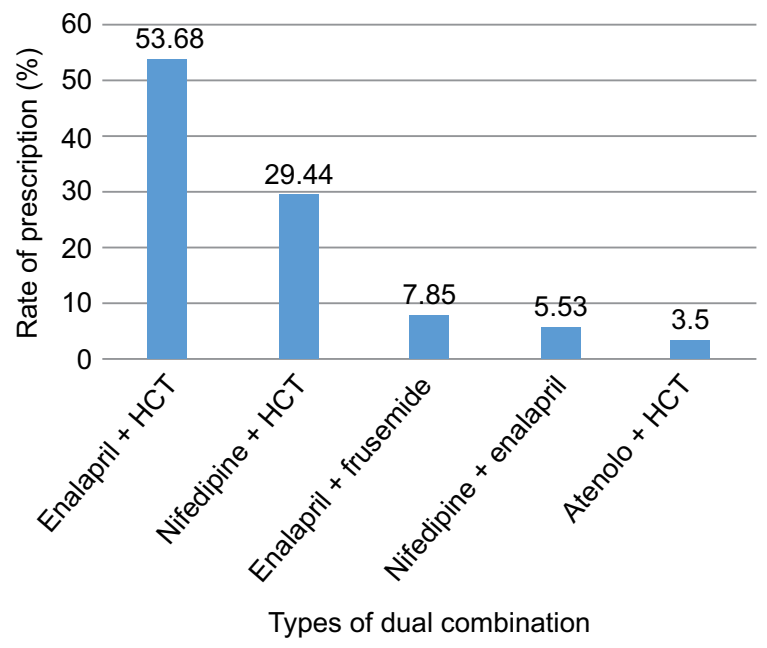

Figure 2 Most commonly prescribed dual therapies.

Abbreviation: HCT, hydrochlothiazide.

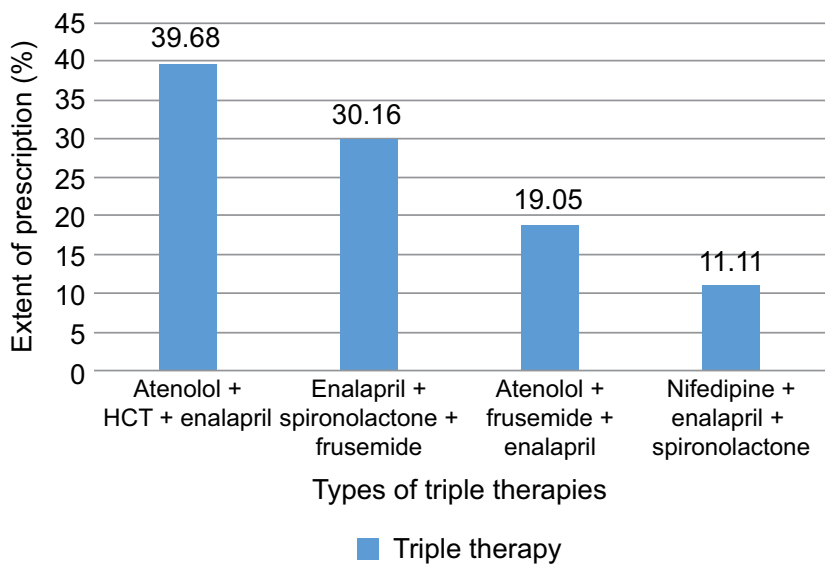

Figure 3 The types of triple therapies.

Abbreviation: HCT, hydrochlothiazide.

\section{Correlation between prescription patterns and rate of blood control}

A binary logistic regression analysis indicated that twicedaily dosing was associated with lower risk of uncontrolled HTN (crude odds ratio [COR] $=0.51[0.15-0.73]$, adjusted odds ratio $[\mathrm{AOR}]=0.69[0.163-0.91]$ ). A duration of one month between appointments was associated with nearly $90 \%$ reduced incidence of uncontrolled BP (COR $=0.15[0.04-0.5], \mathrm{AOR}=0.093[0.024-0.359])$. The prescription of antihypertensive medications based on the standard CPGs was correlated with low occurrence of elevated BP $(\mathrm{COR}=0.356[0.21-0.61], \mathrm{AOR}=0.452[0.23-0.88])$. Frequent regimen changes were found to decrease the rate of elevated BP $(\mathrm{COR}=0.21[0.12-0.37], \mathrm{AOR}=0.71[0.14-0.8])$. The frequency of UBP was reduced by the presence of normal organ functions (COR $=0.98[0.43-2.24]$, AOR $=0.631[0.225-0.810])$. However, adverse drug reactions and dose and drug interactions did not affect the level of BP (Table 3).

\section{Discussion}

$\mathrm{BP}$ control requires the provision of appropriate medications at proper dose, frequency, and duration. Evaluating the current antihypertensive medication prescription practice with respect to the standard guidelines will help to ensure adequate BP control. The current study has found that multiple prescription patterns affected the level of BP. Twice-daily dosing was associated with nearly thirty percent reduction in the risk of BP control compared to once-daily dosing. About $85 \%$ of patients were being prescribed twice-daily doses of drugs. Twice-daily dosing seems fairly easy to remember and can be taken during the night and morning time when people are at home. ${ }^{19} \mathrm{In}$ contrast, once-daily dosing is not easy to track, thus reducing patients' compliance to medication. ${ }^{19,20}$ The selection of the 
Table 3 Relationship between prescription patterns and the level of BP control: a binary logistic regression

\begin{tabular}{|c|c|c|c|c|}
\hline \multirow[t]{2}{*}{ Factors } & \multicolumn{2}{|l|}{ Treatment outcome } & \multirow[t]{2}{*}{ Crude OR [95\%] } & \multirow[t]{2}{*}{ Adjusted OR [95\%] } \\
\hline & Controlled BP, n (\%) & Uncontrolled BP, n (\%) & & \\
\hline \multicolumn{5}{|l|}{ Dose } \\
\hline Low & $27(4.8 I)$ & $57(10.16)$ & $0.157[0.11-1.743]$ & $0.144[0.023-1.089]$ \\
\hline Normal & $35 I(62.56)$ & $105(18.73)$ & I.097[0.247-4.87] & $1.514[0.28-8.15]$ \\
\hline High & $16(2.85)$ & $5(0.89)$ & I & I \\
\hline \multicolumn{5}{|l|}{ Frequency } \\
\hline$\geq 3$ times daily & $2 \mathrm{I}(3.74)$ & 19(3.38) & $0.2 \mid 4[0.05-1.3 \mid]$ & $\mathrm{I} .60[0.025-1.834]$ \\
\hline Two times daily & $335(59.72)$ & $140(24.96)$ & $0.5 \mid[0.15-0.73]^{\mathrm{a}}$ & $0.69[0.163-0.91]^{\mathrm{a}}$ \\
\hline$\leq$ Once daily & $38(6.77)$ & $8(1.43)$ & 1 & 1 \\
\hline \multicolumn{5}{|c|}{ Duration between appointments } \\
\hline Below I month & $43(7.65)$ & $6(1.10)$ & $0.987[0.16-6.11]$ & $0.591[0.079-4.394]$ \\
\hline I Month & $\mid 33(23.7 \mid)$ & $110(19.61)$ & $0.15[0.04-0.5]^{\mathrm{a}}$ & $0.093[0.024-0.359]^{\mathrm{b}}$ \\
\hline 2 Months & $154(27.45)$ & $43(7.64)$ & $0.44[0.126-1.57]$ & $0.440[0.11-1.796]$ \\
\hline 3 Months & $64(11.4 I)$ & $8(1.43)$ & & I \\
\hline \multicolumn{5}{|c|}{ Drug interaction } \\
\hline No & $284(50.62)$ & $91(16.22)$ & $0.95[0.38-1.3]$ & I.39[0.775-I.367] \\
\hline Minor & $26(4.63)$ & II(I.96) & $2.3 I[0.67-7.9]$ & $0.7 \mid[0.974-9.52 I]$ \\
\hline Moderate & $53(9.45)$ & $35(6.24)$ & I.42[0.58-3.49] & $3.317[0.93-11.85 \mid]$ \\
\hline Major & $3 I(5.53)$ & $30(5.35)$ & 1 & 1 \\
\hline \multicolumn{5}{|c|}{ Adherence to practice guidelines } \\
\hline Concordant & $283(50.45)$ & $92(16.39)$ & $0.356[0.2 \mathrm{I}-0.6 \mathrm{I}]^{\mathrm{b}}$ & $0.452[0.23-0.88]^{a}$ \\
\hline Discordant & III(19.79) & $75(13.37)$ & 1 & I \\
\hline \multicolumn{5}{|c|}{ Regimen change } \\
\hline Yes & $49(8.73)$ & $67(11.94)$ & $0.21[0.12-0.37]^{\mathrm{a}}$ & $0.7 \mid[0.14-0.8]^{\mathrm{b}}$ \\
\hline No & $345(61.50)$ & $100(17.83)$ & 1 & $\mathrm{I}$ \\
\hline \multicolumn{5}{|l|}{ Electrolyte test } \\
\hline Normal & $154(27.45)$ & $70(12.48)$ & $0.38[0.08-1.65]$ & $0.860[0.167-4.425]$ \\
\hline Abnormal & $57(10.16)$ & $5(0.89)$ & $0.37[0.08-1.62]$ & $3.41[0.583-19.98]$ \\
\hline Not done & $183(32.63)$ & $92(16.39)$ & 1 & 1 \\
\hline \multicolumn{5}{|c|}{ Organ function test } \\
\hline Normal & $\mid 33(23.7 \mid)$ & $67(11.95)$ & $0.98[0.43-2.24]$ & $0.631[0.225-0.810]^{\mathrm{a}}$ \\
\hline Abnormal & $57(10.16)$ & $22(3.92)$ & $0.74[0.3|-| .7 I]$ & $0.916[0.204-1.86]$ \\
\hline Not done & $204(36.36)$ & $78(13.90)$ & I & I \\
\hline \multicolumn{5}{|c|}{ Adverse drug reaction } \\
\hline Yes & $52(9.27)$ & $43(7.66)$ & $2.3[0.23-4.32]$ & $1.785[0.76-4.192]$ \\
\hline No & $342(60.96)$ & $124(22.11)$ & I & I \\
\hline
\end{tabular}

Notes: aSignificant at 0.05 level, bsignificant at 0.00 I level.

Abbreviations: BP, blood pressure; OR, odds ratio.

ideal frequency of dosing depends on the mutual relationship between the patient and the physician and availability of suitable alternative drugs with different half-life..$^{21,22}$

Fixed monthly appointments were related with low risk of UBP - nearly double the reduction of the risk of UBP. About $43.4 \%$ of patients had made an appointment to return to the clinic in 1 month. People usually remember their date of appointments by fixing the date with certain holidays (often associated with religious holidays). These holidays fall every month. Hence, the low rate of UBP in these patient groups could be attributed to better adherence to date of appointment. But some individuals prefer the long gaps between appointments so as to minimize transport cost. These patients could have completed their medication course prior to the appointment date, thereby increasing their risk for UBP.
Moreover, the dose adjustment and regimen changes based on organ function, tolerance, effectiveness, and weight change is not flexible as these patients rarely undergo laboratory investigations. As evidenced in the present study, periodical regimen changes were associated with reduced risk of elevated BP. The issue of compliance to appointment is very important, especially in developing countries such as Ethiopia where reminders are not possible. The proximity of public health centers also affects the duration and compliance of appointment in low-income countries. It was also noted that the severity of the disease state could be a determinant because patients might not tend to seek medical care once the acute phase of disease has been surpassed. ${ }^{21,23}$

Drug selection based on standard CPGs was linked with more than $50 \%$ reduction in the rate of UBP. Nearly 
two-thirds $(66.8 \%)$ of the respondents were treated per the CPG. The drug selection criteria take into account the patients' medical condition including presence of comorbid illnesses, level of organ function, and age. Older patients usually respond to calcium channel blockers. Patients with DM, chronic kidney disease, and heart failure usually require ACEIs to promote regression and prevent progression of theses comorbidities. ${ }^{24}$ The 2014 JNC-8 HTN guidelines reduce the proportion of adults eligible for drug therapy as it elevates the BP goals for $\geq 60$ years old adults and patients with DM and chronic kidney disease. The guideline seems to be conservative toward the provision of antihypertensive medications. However, based on the present study finding, the use of these guidelines was found to be associated with good control of BP. Another study also indicated that adherence to standard treatment guidelines was found to be related with optimal control of BP. ${ }^{25}$ Unlike this, a study conducted on Korean adults showed that adults who no longer qualified for antihypertensive therapy under the 2014 guidelines had a significantly higher risk of cardiovascular events and poor BP control compared with normotensive adults. ${ }^{26}$

The investigation of organ functions prior to initiation of antihypertensive medications reduced the risk of uncontrolled BP by approximately $40 \%$ compared to no organ function test before prescription. The functioning of drug metabolizing and excreting organs determines the type and amount of drug prescribed for individual patients. Measurement of organ function will help to reduce adverse drug reactions and further organ damage due to toxic drug accumulation. ${ }^{27,28}$ In the present study, thiazide diuretics and ACEIs were the primary antihypertensive medications in both monotherapy and dual therapy. Patients taking these medications should be screened for kidney function before initiation of therapy. Testing of baseline values of serum creatinine, blood urea nitrogen, and aminotransferase is recommended as disease progression could also contribute to the elevation of these biologic parameters. ${ }^{11}$ Nonetheless, drug dose, drug interaction, and adverse drug reaction were not related to magnitude of BP control.

Of all monotherapies, hydrochlothiazide was most frequently prescribed $(60.2 \%)$ followed by enalapril $(17 \%)$. Oliveira-Filho et $\mathrm{al}^{29}$ found that hydrochlorothiazide and captopril were the top two monotherapies prescribed. The same finding was reported by Shukrala and Gabriel ${ }^{30}$ in east Ethiopia. This might be due to the utilization of the same guidelines that advocate the use of diuretics for both monoand combination therapy. A large cross-sectional study from Turkey ${ }^{31}$ showed that the most frequently used monotherapy was ACEIs, followed by $\beta$-blockers, which is contrary to our finding. This discrepancy could be due to the differences in the patient population between the two countries and the different guidelines used (because European guidelines recommend any class of drug as initial therapy). The accessibility of pharmaceuticals through community and hospital pharmacies could also affect the choice of medications prescribed. A drug utilization study in Mexican subjects reported that the leading medications used were ACEIs (63.8\%). This difference could be due to the presence of more patients with significant comorbidities in Mexico (89.6\%), unlike the present study (28\%), requiring ACEIs. ${ }^{32}$

This study has provided an insight into the prescription patterns of antihypertensive medications with respect to the level of BP control. It will help prescribers to pay more attention for specific factors that affect outcome of BP. But, it has some limitations. For one, it is single-center study. Hence, a nationwide study including primary and secondary health care centers is recommended to make the results more representative. We might also have overestimated the actual rate of IPP as pharmacists might have responded for some IPP after prescription.

\section{Conclusion}

In general, monotherapies were frequently prescribed regimens followed by dual and triple therapies, respectively. Diuretics were the monotherapy of choice, whereas their combination with ACEIs was the most commonly prescribed dual therapy. Twice-daily dosing, fixed monthly appointment, prescription based on CPGs, normal organ function test, and regimen changes were associated with low incidence of UBP. However, adverse drug reactions, electrolyte test, amount of dose, and drug interactions did not affect the level of BP. Clinicians should stratify patients based on risk for organ failure and investigate the organ functions of these patients prior to initiation of therapy. This should be done based on the pharmacokinetic profile of antihypertensive medications. The appointment date is better if arranged in accordance with patients' preference and cultural perspectives so as to encourage continuous return to clinic. Clinicians should also always be vigilant and consider regimen changes if the contemporary regimens do not bring about changes in BP; this is because therapeutic modifications could bring about an improvement in BP.

\section{Acknowledgments}

The authors acknowledge University of Gondar for allowing the conduction of this research. Our deepest thanks also go to the physicians and nurses working in the chronic illness ward of Gondar University Hospital. 


\section{Disclosure}

The authors report no conflicts of interest in this work.

\section{References}

1. Tight blood pressure control and risk of macrovascular and microvascular complications in type 2 diabetes: UKPDS 38. UK Prospective Diabetes Study Group. BMJ. 1998;317(7160):703-713.

2. Musini VM, Nazer M, Bassett K, Wright JM. Blood pressure-lowering efficacy of monotherapy with thiazide diuretics for primary hypertension. Cochrane Database Syst Rev. 2014;5:CD003824.

3. James PA, Oparil S, Carter BL, et al. 2014 evidence-based guideline for the management of high blood pressure in adults: report from the panel members appointed to the Eighth Joint National Committee (JNC 8). JAMA. 2014;311(5):507-520.

4. Shrank WH, Hoang T, Ettner SL, et al. The implications of choice: prescribing generic or preferred pharmaceuticals improves medication adherence for chronic conditions. Arch Intern Med. 2006;166(3): 332-337.

5. Mao W, Vu H, Xie Z, Chen W, Tang S. Systematic review on irrational use of medicines in China and Vietnam. PLoS One. 2015;10(3):e0117710.

6. Iyalomhe GB, Omogbai EK, Iyalomhe OO. Long-term electrolyte effects during initiation of antihypertensive therapy with amlodipine or hydrochlorothiazide in diabetic Nigerians. Am J Med Sci Med. 2013;1(3):31-37.

7. Simoens S, Sinnaeve PR. Patient co-payment and adherence to statins: a review and case studies. Cardiovasc Drugs Ther. 2014;28(1): 99-109.

8. Ruberton PM, Huynh HP, Miller TA, Kruse E, Chancellor J, Lyubomirsky $\mathrm{S}$. The relationship between physician humility, physicianpatient communication, and patient health. Patient Educ Couns. 2016;99(7):1138-1145.

9. Mascarenhas OA, Cardozo LJ, Afonso NM, et al. Hypothesized predictors of patient-physician trust and distrust in the elderly: implications for health and disease management. Clin Interv Aging. 2006;1(2):175.

10. Desalegn AA. Assessment of drug use pattern using WHO prescribing indicators at Hawassa University teaching and referral hospital, South Ethiopia: a cross-sectional study. BMC Health Serv Res. 2013; 13(1):1.

11. Borrelli S, De Nicola L, Stanzione G, Conte G, Minutolo R. Resistant hypertension in nondialysis chronic kidney disease. Int $J$ Hypertens. 2013;2013:929183.

12. Chiatti C, Bustacchini S, Furneri G, et al. The economic burden of inappropriate drug prescribing, lack of adherence and compliance, adverse drug events in older people. Drug Saf. 2012;35(1):73-87.

13. Lukali V, Michelo C. Factors associated with irrational drug use at a district hospital in Zambia: patient record-based observations. Med $J$ Zambia. 2015;42(1):25-30.

14. Hailu W, Tamiru W, Berhane A, Tsega B, Ergetie Z, Admassie E. Prescription pattern of psychotropic, narcotic and anticonvulsant drugs in gondar university hospital, north west Ethiopia. Int J Pharm Ind Res. 2012;2(04):424-428.

15. Sebsibe Teni F, Shemsu Surur A, Getie A, Alemseged A, Meselu M. Medication prescribing pattern at a pediatric ward of an Ethiopian hospital. Int J Pediatr. 2014;2(42):23-30.
16. Karimi A, Haerizadeh M, Soleymani F, Haerizadeh M, Taheri F. Evaluation of medicine prescription pattern using World Health Organization prescribing indicators in Iran: a cross-sectional study. J Res Pharm Pract. 2014;3(2):39-45.

17. Food, Medicine and Healthcare Administration and Control Authority of Ethiopia. Ethiopian Standard Treatment Guidelines for General Hospital, 3rd ed. Addis Ababa, Ethiopia: FMHACA of Ethiopia; 2014:47-54.

18. Ushadevi K, Rubiya S, Vigneshwaran E, Padmanabha Y. Drug use evaluation of antihypertensive medications in outpatients in a secondary care hospital. Asian J Pharm Clin Res. 2013;6(2):0974-2441.

19. Hermida RC, Ayala DE, Mojón A, Fernández JR. Bedtime dosing of antihypertensive medications reduces cardiovascular risk in CKD. J Am Soc Nephrol. 2011:22(12):2313-2321.

20. Goyal D, Chong A, Watson R, Prasad N, Watson R. Assessment of single versus twice daily dosing of ramipril by ambulatory blood pressure monitoring in patients similar to those included in the HOPE study. J Hum Hypertens. 2007;21(7):525-530

21. Pacheco RR, Álvarez JN, López FC, et al. Non-compliance with appointments amongst patients attending an allergology clinic, after implementation of an improvement plan. Allergol Immunopathol. 2007;35(4):136-144.

22. Abdalwahed S. Physician adherence to hypertension treatment guidelines and drug acquisition costs of antihypertensive drugs at the cardiac clinic: a pilot study. Patient Prefer Adherence. 2012;6:101-108.

23. Gupta D, Denton B. Appointment scheduling in health care: challenges and opportunities. IIE Transactions. 2008;40(9):800-819.

24. Baltatzi M, Savopoulos C, Hatzitolios A. Role of angiotensin converting enzyme inhibitors and angiotensin receptor blockers in hypertension of chronic kidney disease and renoprotection. Study results. Hippokratia. 2011;15(Suppl 1):27.

25. Rowan CG, Turner JR, Shah A, Spaeder JA. Antihypertensive treatment and blood pressure control relative to hypertension treatment guidelines. Pharmacoepidemiol Drug Saf. 2014;23(12):1294-1302.

26. Ko MJ, Park CM, Kim YJ, Kang SH, Park DW. Clinical application and potential effects of 2014 hypertension guidelines on incident cardiovascular events. Am Heart J. 2015;170(5):1042-1049. e5.

27. Alahdal AM, Elberry AA. Evaluation of applying drug dose adjustment by physicians in patients with renal impairment. Saudi Pharm J. 2012;20(3):217-220.

28. Karsch-Völk M, Schmid E, Wagenpfeil S, Linde K, Heemann U, Schneider A. Kidney function and clinical recommendations of drug dose adjustment in geriatric patients. BMC Geriatr. 2013;13(1):1.

29. Oliveira-Filho AD, Barreto-Filho JA, Neves SJ, Lyra Junior DP. Association between the 8-item Morisky Medication Adherence Scale (MMAS-8) and blood pressure control. Arq Bras Cardiol. 2012;99(1):649-658.

30. Shukrala F, Gabriel T. Assessment of prescribing, dispensing, and patient use pattern of antihypertensive drugs for patients attending outpatient department of Hiwot Fana Specialized University Hospital, Harar, Eastern Ethiopia. Drug Des Devel Ther. 2015;9:519-523.

31. Abaci A, Kozan O, Oguz A, et al. Prescribing pattern of antihypertensive drugs in primary care units in Turkey: results from the TURKSAHA study. Eur J Clin Pharmacol. 2007;63(4):397-402.

32. Alba-Leonel A, Carvajal A, Fierro I, et al. Prescription patterns of antihypertensives in a community-health centre in Mexico City: a drug utilization study. Fundam Clin Pharmacol. 2016;30(3):276-281.
Integrated Pharmacy Research and Practice

\section{Publish your work in this journal}

Integrated Pharmacy Research and Practice is an international, peer-reviewed, open access, online journal, publishing original research, reports, reviews and commentaries on all areas of academic and professional pharmacy practice. This journal aims to represent the academic output of pharmacists and pharmacy practice with particular focus on integrated care. All papers are carefully

\section{Dovepress}

peer reviewed to ensure the highest standards as well as ensuring that we are informing and stimulating pharmaceutical professionals. The manuscript management system is completely online and includes a very quick and fair peer-review system, which is all easy to use. Visit http://www.dovepress.com/ testimonials.php to read real quotes from published authors. 